\title{
Macular Morphology and Visual Acuity in the Comparison of Age-related Macular Degeneration Treatments Trials (CATT)
}

\author{
Glenn J. Jaffe, MD1, Daniel F. Martin, MD², Cynthia A Toth, MD1, Ebenezer Daniel, MPH, \\ $\mathrm{PhD}^{3}$, Maureen G. Maguire, $\mathrm{PhD}^{3}$, Gui-Shuang Ying, $\mathrm{PhD}^{3}$, Juan E. Grunwald, $\mathbf{M D}^{3}$, and \\ Jiayan Huang, MS $^{3}$ for the Comparison of Age-related Macular Degeneration Treatments \\ Trials (CATT) Research Group ${ }^{\star}$ \\ ${ }^{1}$ Department of Ophthalmology, Duke University, Durham, NC \\ ${ }^{2}$ Cole Eye Institute, Cleveland Clinic, Cleveland, $\mathrm{OH}$ \\ ${ }^{3}$ Department of Ophthalmology, University of Pennsylvania, Philadelphia, PA
}

\begin{abstract}
Objective-To describe effects of treatment for one year with ranibizumab or bevacizumab on macular morphology and the association of macular morphology with visual acuity in eyes with neovascular age related macular degeneration (AMD).

Design-Prospective cohort study within a randomized clinical trial.

Participants-Participants in the Comparison of Age-related Macular Degeneration Treatments Trials.

Methods-Participants were assigned randomly to treatment with ranibizumab or bevacizumab on a monthly or as needed schedule. Optical coherence tomography (OCT), fluorescein angiography (FA), color fundus photography (FP), and visual acuity (VA) testing were performed periodically through 52 weeks. Masked readers graded images. General linear models were applied to evaluate effects of time and treatment on outcomes.
\end{abstract}

Main Outcome Measures-Fluid type and location, and thickness by OCT, size and lesion composition on FP and FA, and VA.

Results-Intraretinal fluid (IRF), subretinal fluid (SRF), and sub-retinal pigment epithelium fluid, and retinal, subretinal and subretinal tissue complex thickness decreased in all treatment groups. A higher proportion of eyes treated monthly with ranibizumab had fluid resolution at 4

\footnotetext{
(C) 2013 American Academy of Ophthalmology, Inc. Published by Elsevier Inc. All rights reserved.

* The credit roster for the CATT Research Group is provided in the Appendix 1, available at http://aaojournal.org

Publisher's Disclaimer: This is a PDF file of an unedited manuscript that has been accepted for publication. As a service to our customers we are providing this early version of the manuscript. The manuscript will undergo copyediting, typesetting, and review of the resulting proof before it is published in its final citable form. Please note that during the production process errors may be discovered which could affect the content, and all legal disclaimers that apply to the journal pertain.

This article contains online-only material. The following should appear online-only: Appendix 1.

Financial disclosure: Glenn Jaffe, MD has a consultancy relationship with Heidelberg Engineering and active or pending grants from Regeneron. Cynthia Toth, MD has a consultancy relationship with Physical Sciences Incorporated, active or pending grants from Genentech, Bioptigen, and Physical Sciences Incorporated, a patent pending for OCT analysis technology related to analysis for agerelated macular degeneration, and royalties from Alcon Laboratories for ophthalmic surgical technologies. Dr. Jaffe's and Dr. Toth's institution receives money for these relationships. The other members of the writing committee have no financial relationships to declare.
}

Presented in part at the Association for Research in Vision and Ophthalmology Meeting, Ft. Lauderdale, Florida, 2012, the Macula Society Meeting, Jerusalem, Israel, 2012, and the American Association of Retinal Specialists, Las Vegas, NV, 2012. 
weeks and the difference persisted through 52 weeks. At 52 weeks, there was little association between the presence of fluid of any type (without regard to fluid location) and the mean VA. However, at all time points, eyes with residual IRF, especially foveal IRF, had worse mean VA ( 9 letters) than those without IRF. Eyes with abnormally thin $(<120 \mathrm{u})$ or thick $(>212 \mathrm{u})$ retinas had worse VA than those with normal thickness (120-212 u). At week 52, eyes with larger neovascular lesions or with foveal scar had worse VA than eyes without these features.

Conclusions-Anti-vascular endothelial growth factor (VEGF) therapy reduced lesion activity and improved VA in all treatment groups. At all time points, eyes with residual IRF had worse VA than those without. Eyes with abnormally thin or thick retinas, with residual large lesions, and with scar also had worse VA. Monthly ranibizumab dosing yielded more eyes with no fluid and an abnormally thin retina, although the long-term significance is unknown. These results have important treatment implications in eyes undergoing anti-VEGF therapy for neovascular AMD.

The anti-vascular endothelial growth factor (VEGF) drugs ranibizumab (Lucentis, Genentech, South San Francisco, CA) and bevacizumab (Avastin, Genentech) are highly effective treatments to preserve visual acuity (VA) among individuals with neovascular agerelated macular degeneration (AMD). ${ }^{1-4}$ Despite the dramatic effects on VA, many of the neovascular lesions continue to leak fluid and increase in size as seen on fluorescein angiograms (FA) and optical coherence tomography (OCT). ${ }^{1-4}$ The associations between macular morphological features and VA following intravitreal anti-VEGF treatment are complex and not well understood. In an attempt to shed light on these associations, we now describe the effect of different anti-VEGF treatment strategies on the activity and composition of choroidal neovascularization (CNV) lesions as determined on OCT, color fundus photographs (FP), and FA, as well as the association of lesion activity and composition with VA outcomes among participants of the Comparison of AMD Treatments Trials (CATT).

\section{METHODS}

\section{Study Population}

Details of the design and methods for CATT have been published previously. ${ }^{3}$ Parameters used to determine the participants' morphologic features at baseline and 52 weeks are summarized here. A total of 1185 subject were enrolled by 43 United States clinical centers between February 2008 and December 2009. Only one eye per subject, the study eye, was treated as a part of the clinical trial. Inclusion criteria included subject age $\geq 50$ years, presence of previously untreated active choroidal neovascularization (CNV) secondary to AMD in the study eye, and VA between 20/25 and 20/320. CNV was considered active when leakage or increased stippling on FA and fluid on time-domain OCT was documented through central image review. Fluid on OCT could be within or below the retina or below the retinal pigment epithelium (RPE). CNV or its sequelae (i.e., pigment epithelium detachment, hemorrhage, blocked fluorescence, macular edema, or fluid) needed to involve the center of the fovea. For the CNV to be considered secondary to AMD, at least one druse $>63 \mu$ needed to be present in either the study eye or fellow eye, or the fellow eye needed to have CNV or geographic atrophy. Participants were assigned randomly with equal probability to one of four treatment groups: (1) ranibizumab monthly; (2) bevacizumab monthly; (3) ranibizumab as needed (pro re nata, PRN); and (4) bevacizumab PRN. The institutional review boards associated with each center approved the study. All participants provided written informed consent.

\section{Study Procedures}

All image readers and visual function examiners were masked to the subject's treatment assignment. Certified technicians following standardized procedures performed time domain 
OCTs on all participants at baseline, and 4, 8, 12, 24, and 52 weeks. Technicians obtained Stratus OCT images in the study eye with the Macular Thickness Map and Fast Macular Thickness Map protocols. ${ }^{5}$ Two certified readers at the CATT OCT Reading Center independently analyzed all scans for morphological characteristics. Readers identified intraretinal fluid, subretinal fluid (SRF), and fluid below the retinal pigment epithelium (sub-RPE fluid) and when fluid was present, noted the location of fluid relative to the foveal center: foveal or sub-foveal (within 500u of the foveal center) or beyond 500u of foveal center. Readers also measured at the foveal center retinal thickness, SRF thickness, and subretinal tissue complex thickness, which included material between the inner retina, or subretinal fluid, if present, and Bruch's membrane. Morphologically, the subretinal tissue complex thickness included material above the RPE, termed subretinal highly reflective material, which comprises CNV (and blood and fibrosis, when present), the RPE, and material under the RPE (when a RPE detachment was present). The sum of the three thickness measurements was termed total thickness ${ }^{6}$. A Senior Reader reconciled any grading disagreements between the initial reader pair.

Certified photographers obtained with standardized procedures stereoscopic FP and FA on all participants at baseline and 52 weeks. ${ }^{7}$ Two certified readers at the CATT Photography Reading Center independently analyzed all photographic images, including FPs and FAs to identify the lesion components or fluid under the foveal center, dye leakage on FA, and neovascular lesion area $\left(\mathrm{mm}^{2}\right)$. The baseline neovascular lesion, denoted total CNV lesion, included CNV and contiguous areas of serous pigment epithelium detachment, scar, hemorrhage and blocked fluorescence. At Week 52, geographic atrophy and RPE tear were also included as lesion components when they were within the baseline neovascular lesion. Two readers adjudicated any grading discrepancies; the Reading Center Principal Investigator determined the final grade when the readers could not come to consensus.

Certified visual function examiners followed a standardized protocol to measure VA on all participants at baseline and Weeks 4, 12, 24, 36 and 52. Examiners performed subjective refraction and tested VA with an electronic testing system. ${ }^{8}$

Eyes assigned to monthly treatment received an injection of their assigned drug, either $0.5 \mathrm{mg}(0.05 \mathrm{ml})$ of ranibizumab or $1.25 \mathrm{mg}(0.05 \mathrm{ml})$ of bevacizumab, approximately every 28 days unless they missed a scheduled visit or developed contraindications to treatment. Eyes assigned to PRN treatment were evaluated approximately every 28 days and treated with their assigned drug when there was fluid on OCT or other signs of active neovascularization. ${ }^{3}$

\section{Data and Statistical Analysis}

Only patients who met all eligibility criteria for the clinical trial $(\mathrm{N}=1142)$ were included in the analysis for this paper. The number of patients with OCTs available for grading was 1116, 1091, 1048, 1015, and 1053 at Weeks 4, 8, 12, 24 and 52, respectively. The number of patients with FPs and FAs available for grading was 1033 at Week 52. In addition, specific features on images could be ungradeable because of insufficient image quality; the percentage of images ungradeable at any particular time was approximately $2 \%$ or less each for OCTs, FPs and FAs.

Thickness measurements $(\mu)$ based on OCT were divided into categories as follows: total thickness: 0-325, 326-425, 425-550, and > 550; retinal thickness: 0-119, 120-212, and $>212$; subretinal fluid thickness: $0,1-25$, and > 25; subretinal tissue complex: 0-75, 76-160, 161-275, and >275. Categories were based on the baseline quartiles except for retinal thickness, which was determined based on the mean of healthy eyes on Stratus OCT measured manually \pm 2 standard deviations. ${ }^{9}$ 
General linear models were used to compare the retinal morphology responses or VA responses among 4 treatment groups, between two drug groups or between two treatment regimens. Time was treated as a continuous variable to assess the retinal morphology responses (presence of OCT fluid and OCT thickness) or VA responses over time. The interactions of morphologic responses and VA with treatment groups, drug groups and regimens were also determined. The relationships between retinal thickness and VA were explored using Locally Weighted Scatterplot Smoothing (LOWESS) plots. ${ }^{10}$ The association of retinal morphology findings from FP, FA or OCT with VA at 1 year was analyzed by multiple regression models, which went through backward elimination processes by only retaining in the model the statistically significant morphology findings. All statistical analyses were performed in SAS V9.2 (SAS Institute Inc., Cary, NC), and two-sided p-values $<0.05$ were considered to be statistically significant.

\section{RESULTS}

\section{OCT Morphological Characteristics Over Time by Treatment Group}

Presence and Type of Fluid on OCT Over Time-At baseline, all eligible eyes had at least one type of fluid, reflecting active CNV. The distribution of each fluid type at baseline was similar among the four treatment groups. (Figure 1, A-D). A high proportion of eyes had each of subretinal fluid (83.8\%) and intraretinal fluid (76.7\%). Sub-RPE fluid was present in approximately half $(53.7 \%)$ of the eyes.

Following anti-VEGF therapy, in all treatment groups, the proportion of eyes with fluid of any type (intraretinal, subretinal, or sub-RPE fluid) decreased markedly (Figure 1A). The largest decrease in the proportion with fluid of any type was observed between baseline and 4 weeks, the time of the first OCT after the initial treatment. There was a further small decrease in the proportion with fluid of any type between 4 and 8 weeks followed by relatively little change through Week 52. Also, for each of the 3 types of fluid, the largest decrease in the proportion with fluid was observed between baseline and 4 weeks (Figure 1, B-D). At 4 weeks, the decline in the proportion of eyes with each type of fluid for all treatment groups combined was largest for subretinal fluid (40.5\%), followed by intraretinal fluid (23.3\%), and then sub-RPE fluid (18.0\%). There was further decrease over time through Week 52, mainly between Week 4 and Week 8 for subretinal fluid $(\mathrm{p}<0.0001)$, subRPE fluid ( $\mathrm{p}=0.0009)$, and intraretinal fluid $(\mathrm{p}=0.06)$.

Although subretinal fluid was the fluid type most commonly seen in all groups at baseline, because of the marked reduction in subretinal fluid at 4 weeks, by Week 52, the proportion of eyes with intraretinal fluid $(48.7 \%)$ was greater than the proportions with subretinal fluid $(31.2 \%)$ or sub-RPE fluid (32.8\%; all $\mathrm{p}<0.0001)$.

\section{Impact over Time of Drug and Dosing Regimen on OCT-Determined Fluid-}

The drug and dosing regimen administered during the first year of the study influenced the changes in the proportion of eyes with fluid of any type (Figure 1A). Ranibizumab resolved fluid of any type more effectively than bevacizumab over the first 4 weeks $(p<0.0001)$; thereafter, the difference between drugs persisted, but did not change significantly over the next 48 weeks. Furthermore, monthly treatment eliminated fluid of any type during the first year more effectively than PRN treatment $(\mathrm{p}=0.002)$. The joint effects of the drug and regimen used during the first year yielded considerable variation in the percentage of eyes at Week 52 with complete fluid resolution. Complete resolution was observed in $44.6 \%$ of eyes treated monthly with ranibizumab, $25.7 \%$ of eyes treated monthly with bevacizumab, $24.5 \%$ of eyes treated PRN with ranibizumab, and $19.8 \%$ of eyes treated PRN with bevacizumab. 
When the proportions of eyes with each of the three fluid types were considered, modest differences in the effects of drug and dosing regimen again emerged (Figure 1, B-D). Ranibizumab better eliminated intraretinal fluid than bevacizumab $(\mathrm{p}=0.001)$; the difference between drugs was less marked for subretinal fluid ( $\mathrm{p}=0.052)$ and sub-RPE fluid $(\mathrm{p}=0.11$ ). The difference in intraretinal fluid resolution between drugs was apparent by Week 4 and then persisted with little change over the next 48 weeks (Figure 1B). In contrast, the difference in the proportion with fluid between eyes treated monthly and eyes treated PRN increased over time during the first year for subretinal $(\mathrm{p}<0.0001)$ and sub-RPE fluid $(p<0.006)$. The difference between dosing regimens on intraretinal fluid did not increase with time $(\mathrm{p}=0.24)$.

\section{Impact Over Time of Drug and Dosing Regimen on OCT-Determined} Thickness Measurements-In all treatment groups, anti-VEGF therapy substantially reduced total thickness (Figure 2A). After a sharp decrease from a mean (standard error [SE]) at baseline of $462(5.5) \mu$ to 319 (4.5) $\mu$ at 4 Weeks, there was additional decrease in total thickness, mainly between Week 4 and 8 . The pattern of decrease over time was similar for retinal, SRF, and subretinal tissue complex thicknesses (Figure 2, B-D).

Eyes treated with ranibizumab had a larger decrease between baseline and Week 4 in mean total thickness than eyes treated with bevacizumab (Figure 2A, $\mathrm{p}=0.002$ ); this initial difference between drugs persisted through Week 52. After Week 4, the difference in mean total thickness between eyes treated monthly and eyes treated PRN increased over time $(\mathrm{p}=0.03$, Figure $2 \mathrm{~A})$. Most of the differences among treatment groups in total thickness were attributable to the effects on the subretinal tissue complex. The mean subretinal tissue complex thickness decreased more between baseline and 4 weeks in eyes treated with ranibizumab $(\mathrm{p}=0.008)$ and the difference persisted through Week 52. Also, decrease in mean thickness of the subretinal tissue complex between Week 4 and 52 was greater for eyes treated monthly than for eyes treated PRN ( $p=0.04$, Figure 2D).

As one would expect, the proportion of eyes with thinner than normal retinas $(<120 \mathrm{u}$, which is greater than 2 standard deviations from the mean Stratus measurement among normal subjects $^{9}$ ) was very small at baseline, and increased progressively in all treatment groups by Week 52 (Figure 3). This effect was more pronounced among the eyes treated with ranibizumab on a monthly basis. In this treatment group, there was a higher proportion of eyes with thinner than normal retinas by Week 52 , when compared to the other treatment groups ( $26.4 \%$ versus $20.2 \%$, respectively, $\mathrm{p}=0.05$ ). At the same time, the number of eyes with thicker than normal retinas, $>212 \mathrm{u}$, decreased markedly between the baseline and Week 52 visits, and the lowest proportion of eyes with thicker than normal retinas was observed in the ranibizumab monthly treatment group (Table 1; Figure 3).

Given the higher proportion of thinner than normal retinas in ranibizumab monthly-treated eyes, we determined whether the difference in retinal thickness depended on residual intraretinal fluid. When one considers only eyes without residual intraretinal fluid at the foveal center, eyes treated with ranibizumab monthly still had a higher proportion of abnormally thin retinas (28.4\%) compared to the other three treatment groups combined (22.3\%), but not to a statistically significant degree $(\mathrm{p}=0.06$; Table 1$)$.

\section{Lesion Components Under the Foveal Center by Drug and Dosing Regimen-} On baseline FA and FP, the majority of eyes (59.2\%) had CNV under the foveal center, $26.8 \%$ had fluid only and an additional $8.0 \%$ had hemorrhage under the foveal center (Figure 4A). After 52 weeks of anti-VEGF therapy, the percentages with CNV under the foveal center decreased substantially to $24.8 \%$ (Figure 4B). Only 3 eyes $(0.3 \%)$ had hemorrhage in the foveal center and the percentage with only fluid decreased to $8.2 \%$. Non- 
geographic atrophy (depigmented RPE without clearly defined boundaries) developed in the foveal center in $14.6 \%$, and scar in $18.6 \%$. There was no foveal center pathology in $19.6 \%$ of eyes; the majority of which had fluid only $(60.1 \%)$ or hemorrhage $(7.4 \%)$ in the foveal center at baseline.

Among the four treatment groups, foveal involvement at baseline was very similar (data not shown). At 52 weeks, the distribution of foveal center CNV and CNV sequelae was generally similar across all treatment groups with no significant drug or dosing regimen effects ( $p>0.07$; Figure 5 A-D).

\section{Correlation of Morphology with VA}

To assess the functional impact of observed morphological changes induced by anti-VEGF therapy, we determined the correlation of VA with OCT and photographic (color fundus photographic and angiographic) morphological parameters, stratified by drug and regimen.

Correlation of Fluid on OCT-with VA-Among all participants, at 52 weeks, there was little association between the presence of fluid of any type (without regard to fluid location) and the mean VA. Furthermore, the mean (standard error [SE]) VA was similar whether fluid was absent (69.7 (1.0) letters), foveal (67.5 (0.8), or extra-foveal (68.4 (1.0)); $\mathrm{p}=0.25$; Figure 6A).

In contrast to results obtained without regard to fluid location, when each fluid location was considered individually, eyes with intraretinal fluid had worse VA than eyes without intraretinal fluid, and eyes with foveal intraretinal fluid had worse VA than eyes with extrafoveal intraretinal fluid. The adverse effect of foveal intraretinal fluid was apparent by 4 weeks, and persisted over time; VA in eyes with foveal intraretinal fluid was nearly two lines worse than in eyes without fluid, and was one line worse than in eyes with extra-foveal intraretinal fluid at all time points evaluated ( $\mathrm{p}<0.0001$; Figure 6). At 52 weeks, mean (SE) VA of eyes with foveal, extrafoveal, and no intraretinal fluid was (62.4 (1.3), (67.2 (1.0), and (71.2 (0.7) letters, respectively ( $\mathrm{p}<0.0001$; Figure 6B). In contrast, at 52 weeks, presence and foveal involvement of sub-RPE fluid and subretinal fluid had little impact on mean VA ( $\mathrm{p}=0.40$ and 0.051 , respectively; Figure 6, C-D).

In a longitudinal model of VA between 4 and 52 weeks that included presence and foveal involvement of each type of fluid as well as total thickness and follow-up time, the strong effect of intraretinal fluid persisted ( $\mathrm{p}<0.0001)$. In contrast, VA was not significantly affected by the presence of subretinal and sub-RPE fluid or total thickness when all of these factors were considered simultaneously.

When the impact of the presence and foveal involvement of intraretinal fluid on VA was assessed over time and among treatment groups, we found no significant interactions between intraretinal fluid and treatment group or with time ( $p>0.05$ for interactions). Taken together, the results indicate that across treatment groups, over time, residual intraretinal fluid, particularly intraretinal foveal fluid, had a significant effect on VA whereas subretinal or sub-RPE fluid did not.

Correlation of OCT-Determined Thickness Measurements with VA-The correlation of VA with thickness at the foveal center depended on the tissue layer (retina, subretinal fluid, and subretinal tissue complex) and time in follow-up. At baseline, when substantial fluid was present, greater total thickness was associated with worse VA for ( $<<0.0001$; Figure 7A). When total thickness was $<325 \mu$, mean (SE) VA was 65.4 (0.7) letters and when total thickness was $>550 \mu$, mean VA was $53.8(0.8)$ letters. Thicker subretinal tissue complex at baseline was also associated with worse VA as was retinal 
thickness $>212 \mu(\mathrm{P}<0.0001$; Figure $7 \mathrm{~B}, \mathrm{D})$. However, the mean VA among eyes with no subretinal fluid (59.2 (0.5) letters), or subretinal fluid $\leq 25 \mu$ (62.4(1.4) letters) was worse than among eyes with $>25 \mu$ subretinal fluid $(63.7(0.7)$ letters; $\mathrm{p}<0.0001)$.

The overall relation of VA to thickness during the 52 weeks after initiation of treatment was explored by combining data from Weeks 4, 12, 24, which were summarized with LOWESS curves (Figure 8). The relation differed by retinal layer. For the retina (Figure 8B), eyes with either low or high retinal thickness had worse VA. Within the range of 120-212 (mean of healthy eyes on Stratus OCT measured manually \pm 2 standard deviations), the LOWESS curve was relatively flat while progressively worse VA was associated with both lower retinal thickness and higher retinal thickness. For subretinal fluid thickness, acuity was lower when there was no fluid present or a relatively thick layer of fluid present (Figure 8C). VA was highest when there was either no or only a thin subretinal tissue complex under the fovea (Figure 8D).

Figure 7 displays the mean VA at different times during follow-up in subgroups of eyes classified by the total central foveal thickness and by thickness of the retinal tissue layers. The trends described above for the combined data generally held for each time point. At all follow-up time points, eyes with retinal thickness between 120 and $212 \mu$ had better VA than eyes with thickness $<120 \mu$ and eyes with thickness $>212 \mu$. By Week 52, the mean VA in eyes with retinal thickness between 120 and $212 \mu$ was 12.4 letters better than in eyes with retinal thickness $<120 \mu$. Eyes with a relatively thin layer of subretinal fluid had a mean VA at 52 weeks that was 5.3 letters better than eyes without any subretinal fluid. Eyes with a subretinal tissue complex thickness of 0 to $75 \mu$ had mean VA at 52 weeks that was 5.1 to 7.1 letters better than the three subretinal tissue complex subgroups with greater thickness.

Because thinner than normal retinas (thickness less than $120 \mathrm{u}$ ) tended to have worse VA than their thicker counterparts, we examined in more detail, the relationship between time to onset of retinal thickness less than $120 \mathrm{u}$ with the visual acuity at 52 weeks. Interestingly, the Week 52 VA depended on the time at which the retina became thinner than normal. Among eyes that ever had a retina thickness $<120 \mathrm{u}$, the best average Week 52 VA (69 letters) was in eyes that were thinner than normal at baseline. When the onset of a thinner retina was later in follow-up, the Week 52 VA was worse with Week 52 VA of 65, 65, 60, and 62 letters thinner retinas first observed at Week 4, 12, 24, and 52 respectively ( $\mathrm{p}=0.01$; for time).

\section{Correlation of Fundus Features determined on Fluorescein Angiograms and Color Fundus Photographs with VA at 52 Weeks-At Week 52, larger neovascular} lesion area was associated with worse VA $(p<0.0001$; Table 2$)$. The eyes with lesion area in the smallest quartile had a mean (SE) VA of 74.3 (1.1) letters while the mean VA among eyes with lesion area in the largest quartile was 61.9 (1.1) letters. The pathology in the foveal center determined by FA and FP also strongly influenced VA ( $<<0.0001$; Table 2). Eyes with no apparent pathology in the foveal center and those with fluid under the foveal center had mean VA of approximately 75 letters, while mean (SE) VA was lowest among eyes with scar (59.5 (1.3) letters) in the foveal center.

\section{Multivariate Analysis of the Association between VA and OCT and Fundus Features at 52 Weeks-When presence and foveal involvement of each of the three} types of fluid on OCT, the thickness of each of the three retinal layers, the lesion size, and foveal pathology were considered simultaneously in a linear regression model of VA (Table 3 ), presence and foveal involvement of intraretinal fluid and subretinal fluid, retinal thickness less than $120 \mu$, larger CNV lesion area, and the type of foveal pathology were independent predictors of VA. In an alternative model with the categories of thickness of 
subretinal tissue complex at the fovea derived from OCT included and the type of foveal pathology derived from FA and FP excluded, the categories of thickness of subretinal tissue complex were a significant predictor of visual acuity $(\mathrm{p}=0.01)$. There was no strong association with either drug or dosing regimen ( $\mathrm{p}=0.24$ and $\mathrm{p}=0.13$, respectively) nor $\mathrm{did}$ drug or dosing regimen affect the association of these factors with VA.

\section{DISCUSSION}

In this study, several morphologic features determined on OCT, FA, and FP were significantly impacted by intravitreal anti-VEGF therapy, and had a significant relationship with VA. In particular, anti-VEGF treatment, regardless of drug and regimen, generally caused rapid and sustained reduction in macular fluid and thickness, and stabilized lesion growth, reduced vascular leakage, and normalized retinal anatomy. Furthermore, presence of intraretinal fluid, abnormally thin or thick retinas, larger CNV area, and foveal scar were associated with the largest decreases in VA at Week 52, when compared to other features evaluated.

A key study finding was that intraretinal fluid (cysts) as determined by OCT had a much greater negative impact on VA than subretinal fluid or sub-RPE fluid at all time points analyzed. In a previous report, eyes with cystoid macular edema, as observed on OCT, associated with sub-foveal CNV had worse VA than those with sub-foveal CNV but no cystoid macular edema. ${ }^{11} \mathrm{We}$ found that intraretinal fluid was associated independently with worse VA, over the entire study duration, when controlling for other potentially confounding variables, even in eyes with sub-foveal CNV. Indeed, sub-foveal CNV did not worsen the adverse effect of intraretinal fluid on VA. The reason for the specific negative impact on VA of intraretinal fluid, but not subretinal fluid or sub-RPE fluid, is not entirely clear. In the beginning of the study, the majority of eyes had cysts, whereas the proportion of eyes with residual intraretinal fluid at Week 52 was much less, and the cysts tended to be small. We hypothesize that early in the study, when the proportion of eyes with intraretinal fluid decreased markedly, the intraretinal fluid was largely driven by VEGF-mediated vascular permeability. However, towards the end of the first year, when intraretinal fluid was eliminated at a much lower rate, we speculate that non-VEGF-mediated mechanisms, such as apoptotic or necrotic cell death accounted for some of the small hyporeflective cystoid structures.

What are the clinical implications of the adverse relationship between intraretinal fluid, particularly foveal intraretinal fluid, and VA? This relationship held at all times during the study, was independent of other morphological features, and was insensitive to the specific anti-VEGF drug and regimen. Based on these data, if one chooses a PRN treatment strategy, we believe that it would be reasonable to aggressively treat intraretinal fluid, particularly when located in the fovea, during the first few months following initiation of therapy in treatment-naïve participants, when intraretinal fluid responds dramatically to therapy, and to continue to treat as long as there is continued intraretinal fluid improvement. In addition, given the favorable outcomes of most participants in whom OCT drove treatment of subretinal and sub-RPE fluid, even, when intraretinal fluid is absent, one should also consider treatment of sub-retinal and sub-RPE fluid, at least early during the course of therapy. In contrast, towards the end of the first year of therapy, aggressive treatment of small amounts of subretinal fluid or sub-RPE fluid, and possibly even intraretinal fluid, that does not change significantly from one exam to the next, may not be warranted. CATT was not designed to address this point and further studies are needed to determine whether there is an adverse VA impact when small amounts fluid that persist after several months of therapy are not treated.

Ophthalmology. Author manuscript; available in PMC 2014 September 01. 
There was a bimodal effect of retinal thickness on VA. As expected, abnormally thick retinas had decreased visual acuities. Furthermore, abnormally thin retinas also had worse VA. Because both geographic and non-geographic atrophy also developed after anti-VEGF therapy, geographic atrophy could be one of the causes of retinal thinning and associated decreased VA. It is unknown, however, whether the observed pathological retinal thinning occurred specifically in regions of underlying RPE and choriocapillaris atrophy. Although it is beyond the scope of this paper, we are currently exploring the relationship between retinal thinning, geographic and non-geographic atrophy and VA. This investigation will be facilitated by a recently completed spectral domain CATT sub-study, in which many more cross sectional images are available to correlate with fundus photographic and fluorescein angiographic features than are available with the 6 radial cross-sectional images from the Stratus OCT machine.

A higher proportion of eyes treated with ranibizumab monthly had abnormally thin retinas $(<120 \mu)$. The precise relationship between drug, dosing, and retinal thinning, however, remains unclear. It is possible that retinal thinning due to loss of neural tissue may occur in all eyes treated with anti-VEGF therapy independent of specific drug and dosing regimen. The differences in retinal thinning could be due in part to the differences in the amount of residual fluid between treatment groups, with greater retinal thickness masking the loss of neural tissue. However, when analysis was restricted to only eyes that had no residual fluid on OCT (as determined in masked fashion by the OCT Reading Center), there was still a higher proportion of eyes with abnormal retinal thinning in eyes treated with ranibizumab monthly as compared to other treatment groups. Additional studies are clearly required to confirm if ranibizumab monthly is truly associated with a higher rate of neural tissue loss.

Thicker sub-foveal subretinal tissue complex, which may include CNV, hemorrhage, fibrosis, and fibrovascular or serous pigment epithelium detachment, was significantly and independently correlated with worse VA. This material is not always given attention in analyses of anti-VEGF effects. ${ }^{12,13}$ However, our data clearly show that in many eyes, antiVEGF therapy reduces this tissue height, as measured by OCT, and decreases components of this tissue, such as subretinal hemorrhage and CNV, as observed on FA and FP. Indeed, in a substantial proportion of eyes, this tissue was completely eliminated by Week 52 . These data, which demonstrate reduction of tissue, in addition to fluid, and the observed slowing of lesion growth, suggest an anti-VEGF therapeutic effect on VA that includes not only reduced vascular permeability, but also regression of pathological tissue. The data further indicate that when ant-VEGF therapy is not fully effective in this regard, visual acuity is negatively impacted.

Foveal lesion composition changed dramatically during the first year of anti-VEGF treatment, and the effect was independent of drug and dosing regimen. In general, the observed effects were desired, with decreased retinal fluid, hemorrhage, and CNV. However, there were some treatment emergent adverse effects, including atrophy, and fibrosis. The latter was associated with the greatest reduction in VA. Ideally, it would be possible to achieve favorable effects on macular anatomy without generating subretinal fibrosis. It would be beneficial in this regard to develop treatments that have an anti-fibrotic effect that might be used in combination with anti-VEGF therapy.

\section{Supplementary Material}

Refer to Web version on PubMed Central for supplementary material. 


\section{Acknowledgments}

Supported by cooperative agreements U10 EY017823, U10 EY017825, U10 EY017826, and U10 EY017828 from the National Eye Institute, National Institutes of Health, Department of Health and Human Services, Bethesda, Maryland. The funding organization participated in the design and conduct of the study and review of the manuscript.

\section{REFERENCES}

1. Brown DM, Kaiser PK, Michels M, et al. ANCHOR Study Group. Ranibizumab versus verteporfin for neovascular age-related macular degeneration. N Engl J Med. 2006; 355:1432-1444. [PubMed: 17021319]

2. Martin DF, Maguire MG, Fine SL, et al. Comparison of Age-related Macular Degeneration Treatments Trials (CATT) Research Group. Ranibizumab and bevacizumab for treatment of neovascular age-related macular degeneration: two-year results. Ophthalmology. 2012; 119:13881398. [PubMed: 22555112]

3. Martin DF, Maguire MG, Ying GS, et al. CATT Research Group. Ranibizumab and bevacizumab for neovascular age-related macular degeneration. N Engl J Med. 2011; 364:1897-1908. [PubMed: 21526923]

4. Rosenfeld PJ, Brown DM, Heier JS, et al. MARINA Study Group. Ranibizumab for neovascular age-related macular degeneration. N Engl J Med. 2006; 355:1419-1431. [PubMed: 17021318]

5. Decroos FC, Toth CA, Stinnett SS, et al. Optical coherence tomography grading reproducibility during the Comparison of Age-Related Macular Degeneration Treatments Trials. Ophthalmology. 2012; 119:2549-2557. [PubMed: 22939114]

6. Ying GS, Huang J, Maguire MG, et al. Comparison of Age-related Macular Degeneration Treatments Trials Research Group. Baseline predictors for one-year visual outcomes with ranibizumab or bevacizumab for neovascular age-related macular degeneration. Ophthalmology. 2013; 120:122-129. [PubMed: 23047002]

7. Grunwald JE, Daniel E, Ying GS, et al. CATT Research Group. Photographic assessment of baseline fundus morphologic features in the Comparison of Age-Related Macular Degeneration Treatments Trials. Ophthalmology. 2012; 119:1634-1641. [PubMed: 22512984]

8. Beck RW, Moke PS, Turpin AH, et al. A computerized method of visual acuity testing: adaptation of the Early Treatment of Diabetic Retinopathy Sstudy testing protocol. Am J Ophthalmol. 2003; 135:194-205. [PubMed: 12566024]

9. Chan A, Duker JS, Ko TH, et al. Normal macular thickness measurements in healthy eyes using Stratus optical coherence tomography. Arch Ophthalmol. 2006; 124:193-198. [PubMed: 16476888]

10. Cleveland WS. Robust locally weighted regression and smoothing scatterplots. J Am Stat Assoc. 1979; 74:829-836.

11. Ting TD, Oh M, Cox TA, et al. Decreased visual acuity associated with cystoid macular edema in neovascular age-related macular degeneration. Arch Ophthalmol. 2002; 120:731-737. [PubMed: 12049577]

12. Gamulescu MA, Radeck V, Lustinger B, et al. Bevacizumab versus ranibizumab in the treatment of exudative age-related macular degeneration. Int Ophthalmol. 2010; 30:261-266. [PubMed: 19633973]

13. Kaiser PK, Blodi BA, Shapiro H, Acharya NR. MARINA Study Group. Angiographic and optical coherence tomographic results of the MARINA Study of ranibizumab in neovascular age-related macular degeneration. Ophthalmology. 2007; 114:1868-1875. [PubMed: 17628683] 
(A) Fluid of Any Type

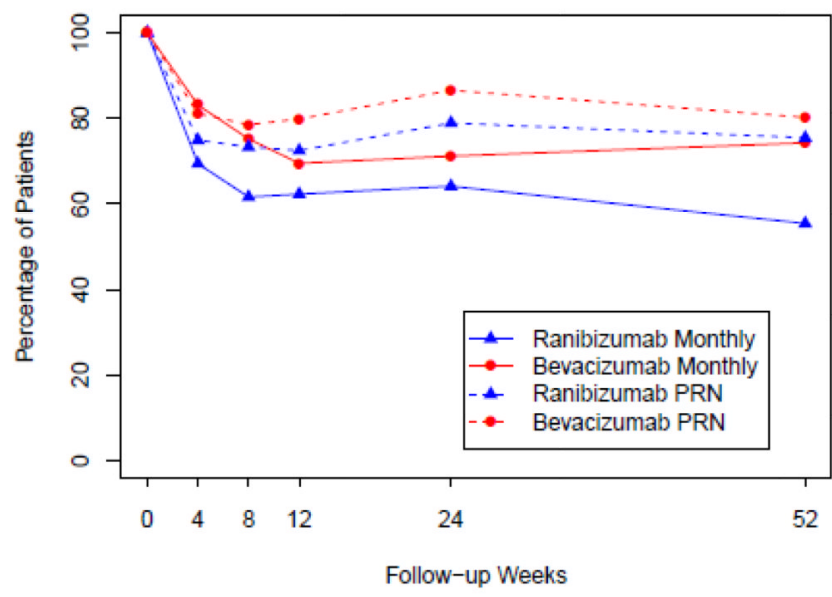

(C) Subretinal Fluid

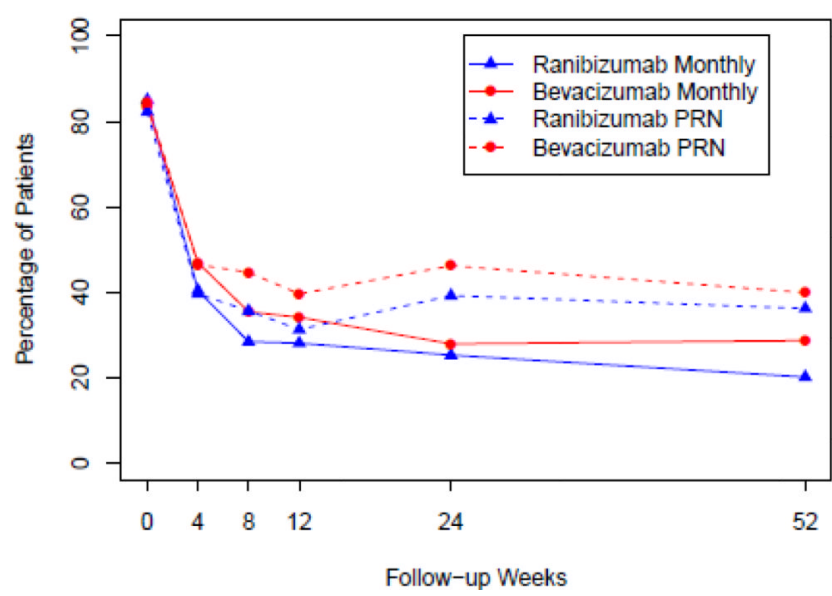

(B) Intraretinal Fluid

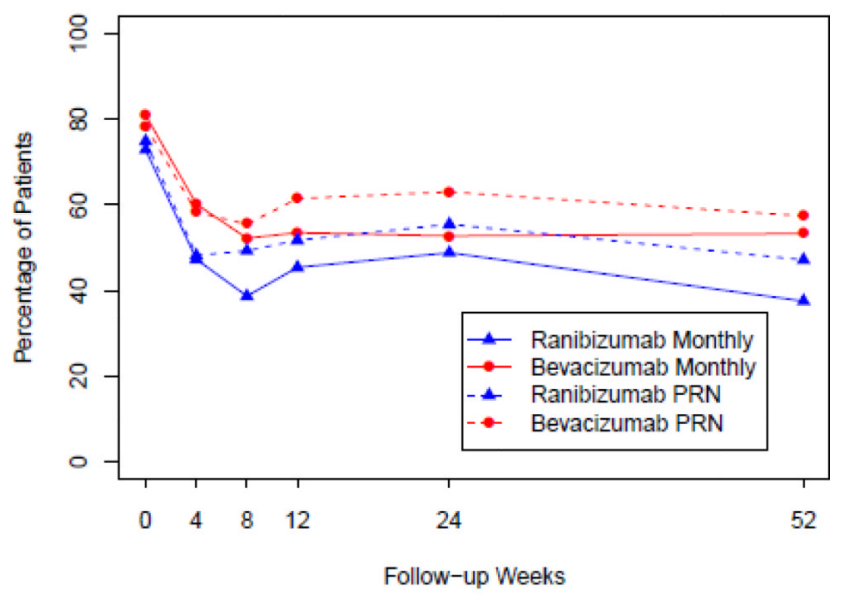

(D) Sub-Retinal Pigment Epithelium Fluid

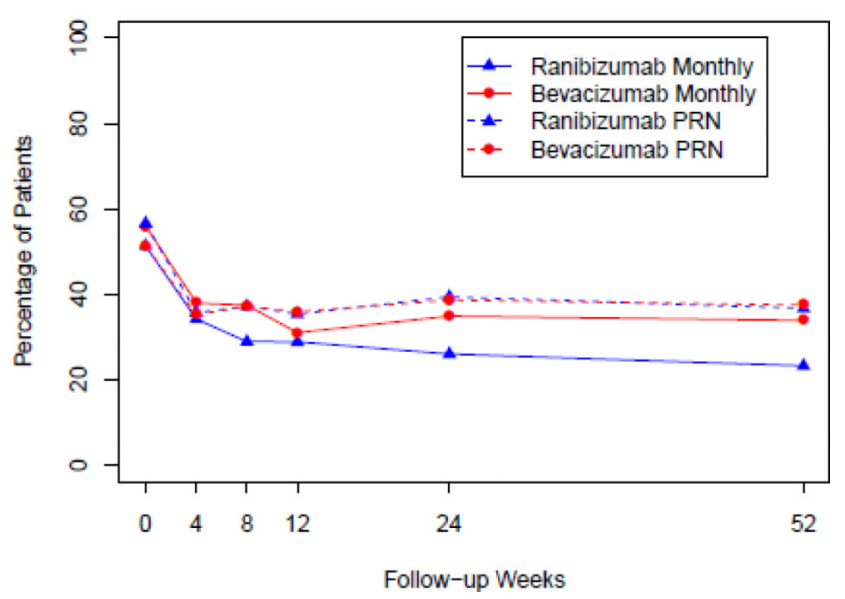

Figure 1.

Percentage in each treatment group over time with (A) fluid of any type, (B) intraretinal fluid, (C) subretinal fluid, (D) sub-retinal pigment epithelium fluid. PRN = pro re nata (as needed). 
(A) Total Thickness

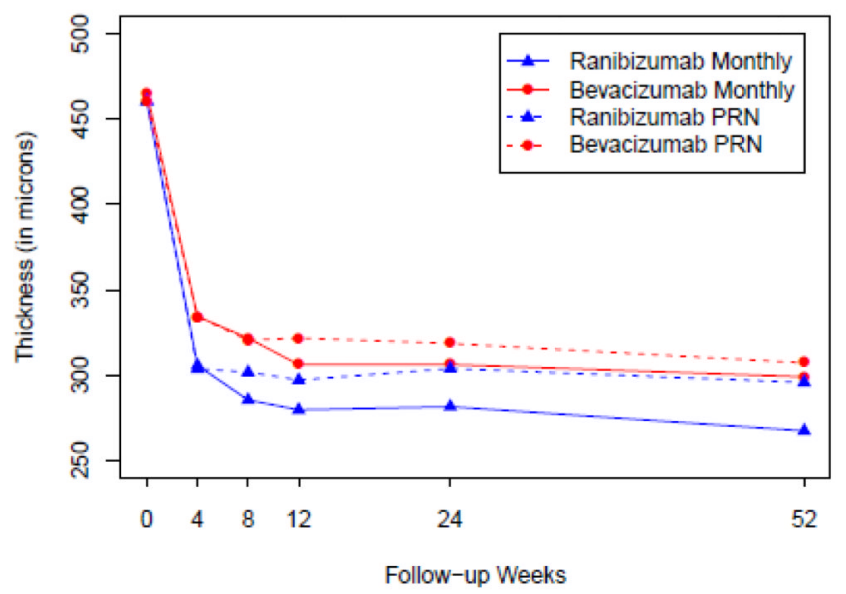

(C) Subretinal Thickness

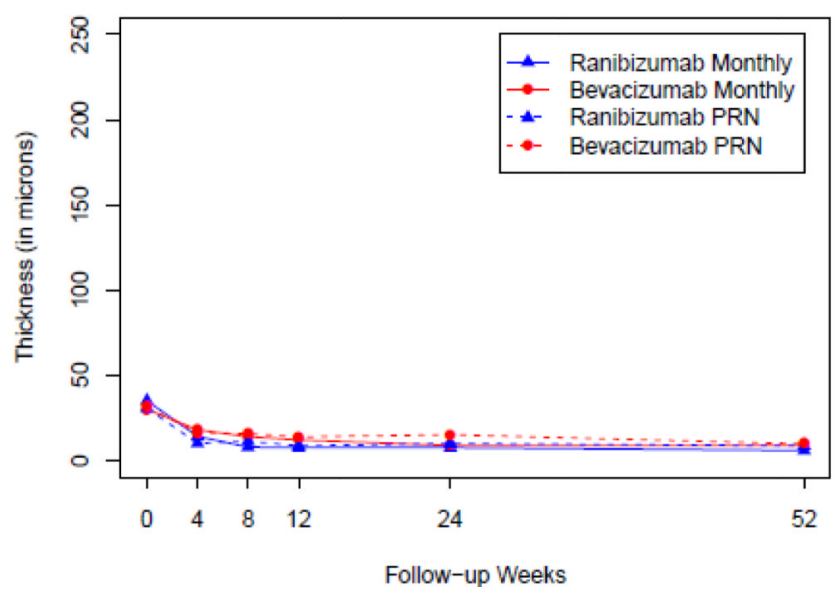

(B) Retinal Thickness

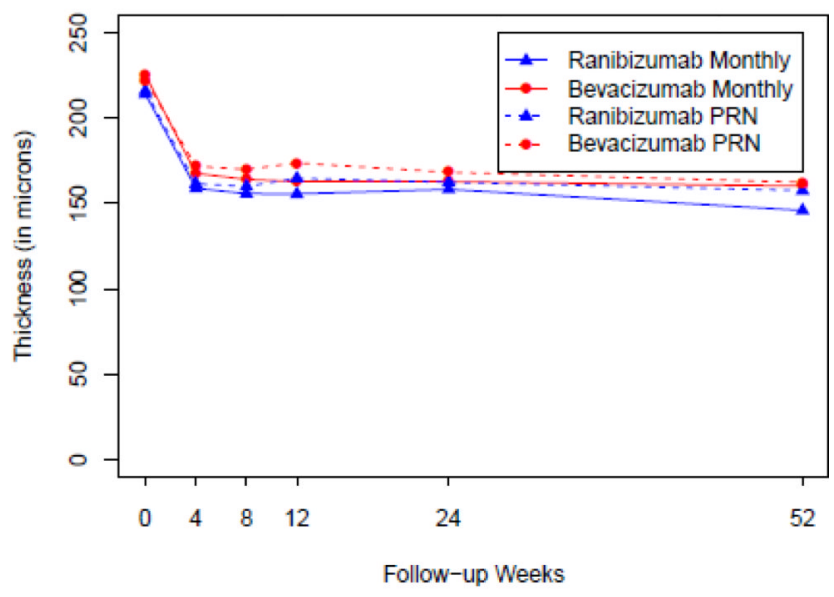

(D) Subretinal Tissue Complex Thickness

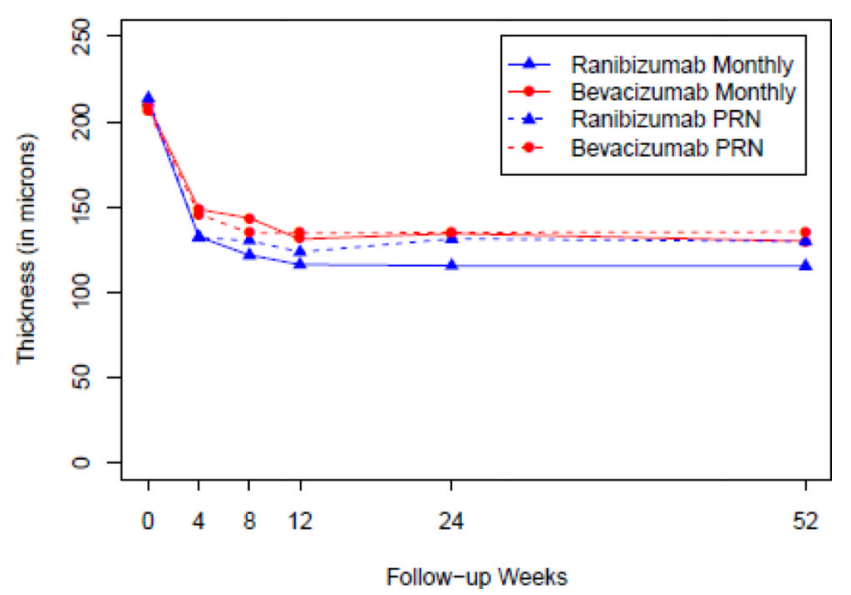

Figure 2.

OCT thickness over time by treatment group for (A) total thickness, (B) retinal thickness, (C) subretinal thickness, (D) subretinal tissue complex thickness. PRN = pro re nata (as needed). 


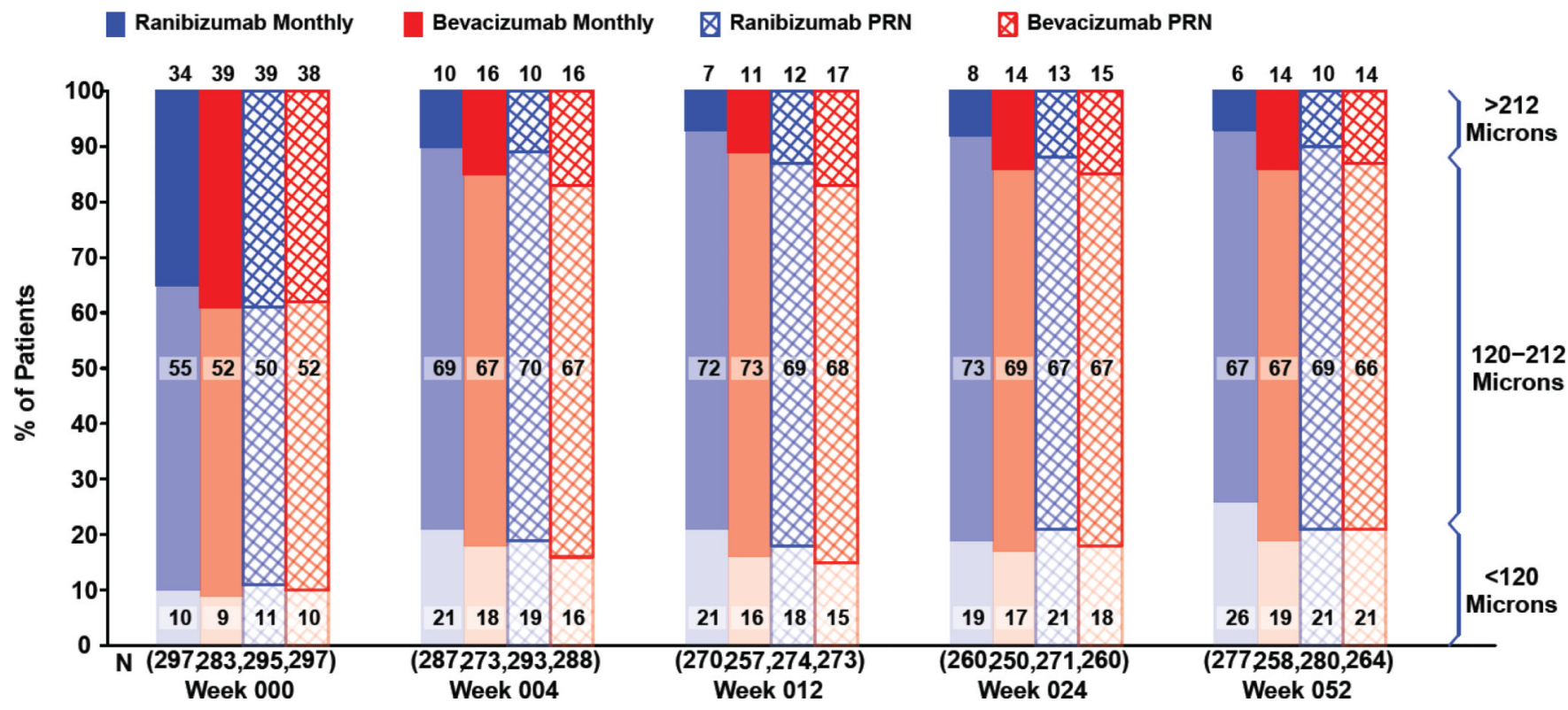

Figure 3.

Retinal thickness category over time by treatment group. PRN = pro re nata (as needed). 


\section{A. Baseline}

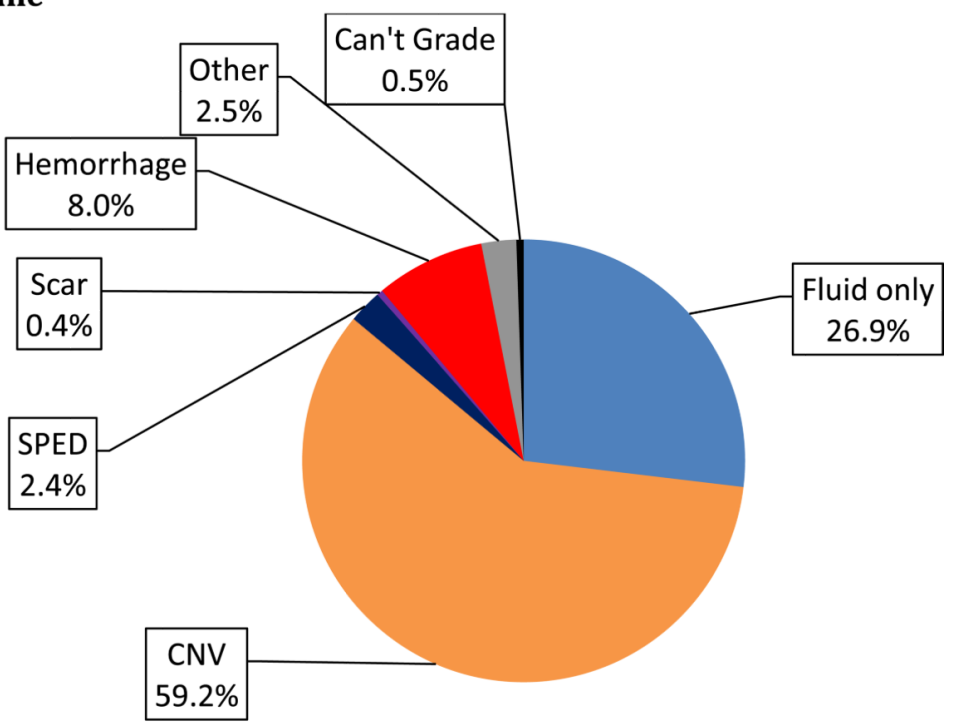

B. Week 52

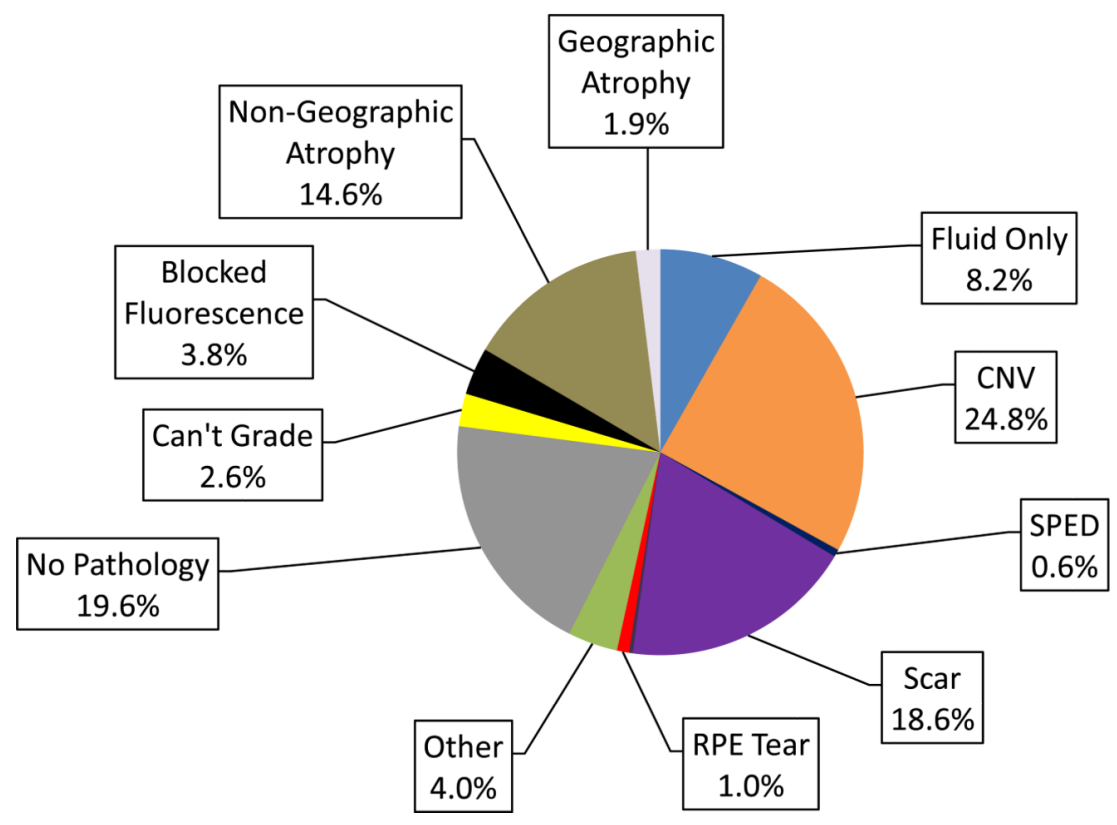

Figure 4.

Involvement of the foveal center by choroidal neovascularization $(\mathrm{CNV})$ or sequelae of $\mathrm{CNV}$ at (A) baseline and (B) Week 52. SPED = serous pigment epithelial detachment. 


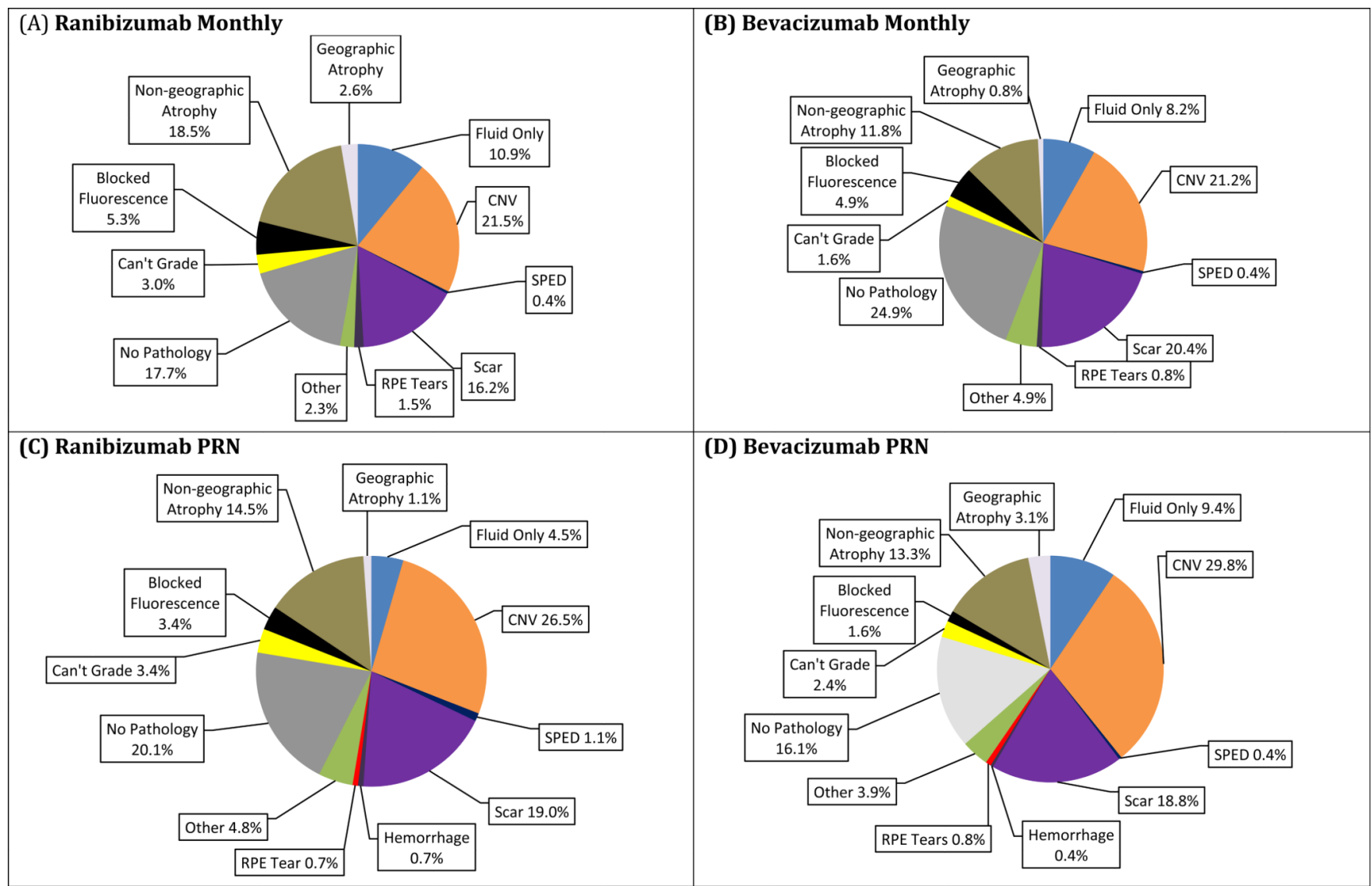

Figure 5.

Involvement of the foveal center by choroidal neovascularization (CNV )or sequelae of CNV at Week 52 for (A) ranibizumab monthly; (B) bevacizumab monthly; (C) ranibizumab PRN; (D) bevacizumab PRN. PRN = pro re nata (as needed) and SPED = serous pigment epithelial detachment. 


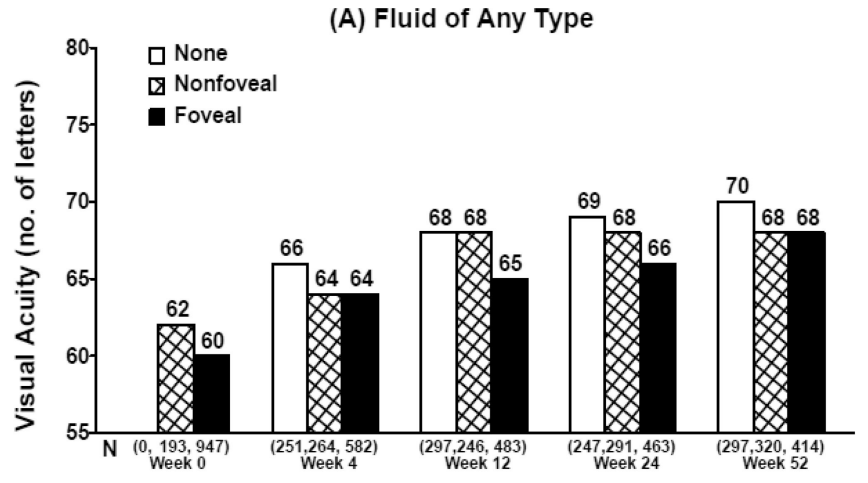

(C) Subretinal Fluid

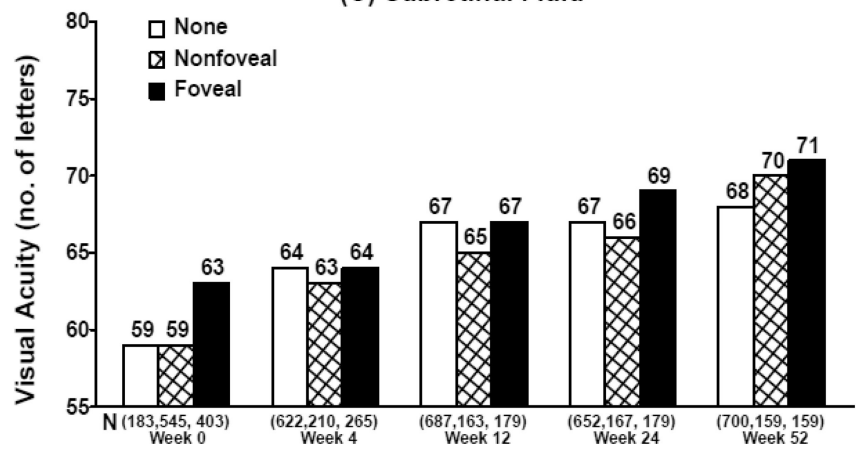

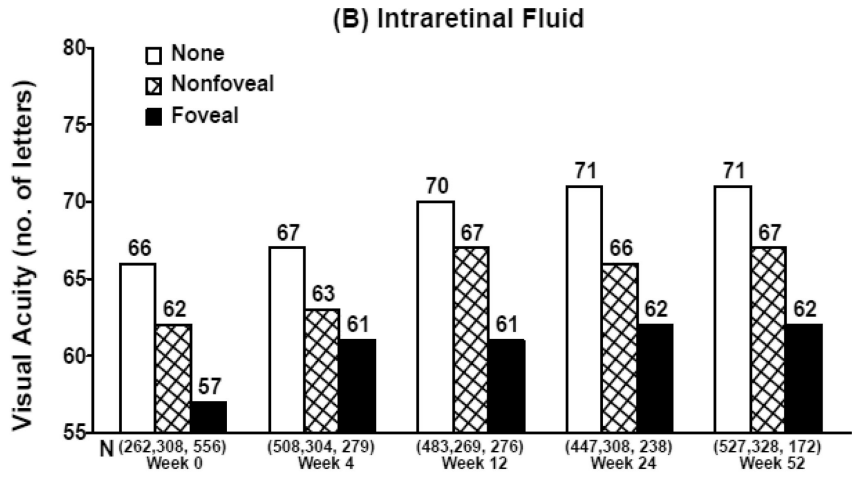

(D) Sub-Retinal Pigment Epithelium Fluid

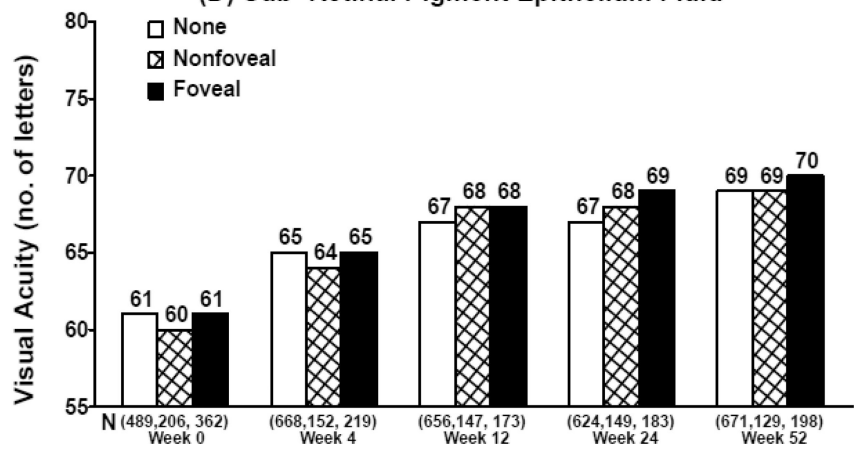

Figure 6.

Mean visual acuity by status of fluid by time for (A) fluid of any type ; (B) intraretinal fluid; (C) subretinal fluid; (D) sub-retinal pigment epithelium fluid. 


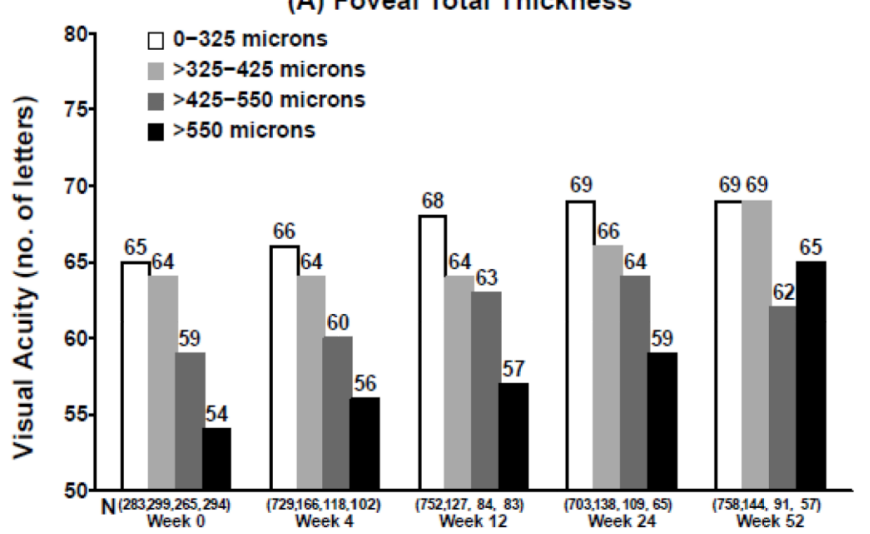

(C) Subretinal Thickness

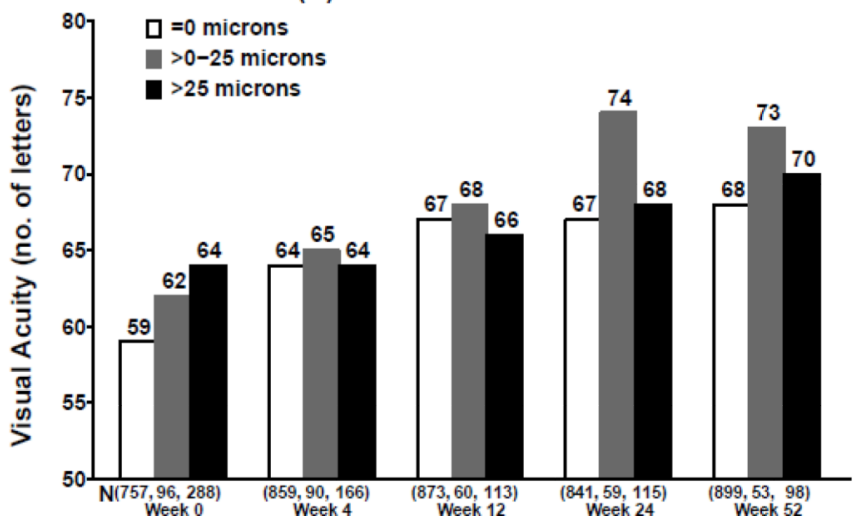

(B) Retinal Thickness

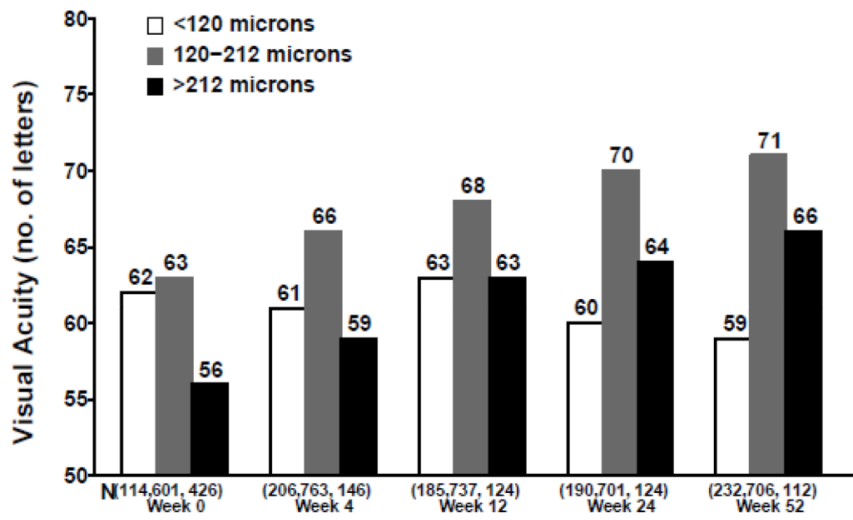

(D) Subretinal Tissue Complex Thickness

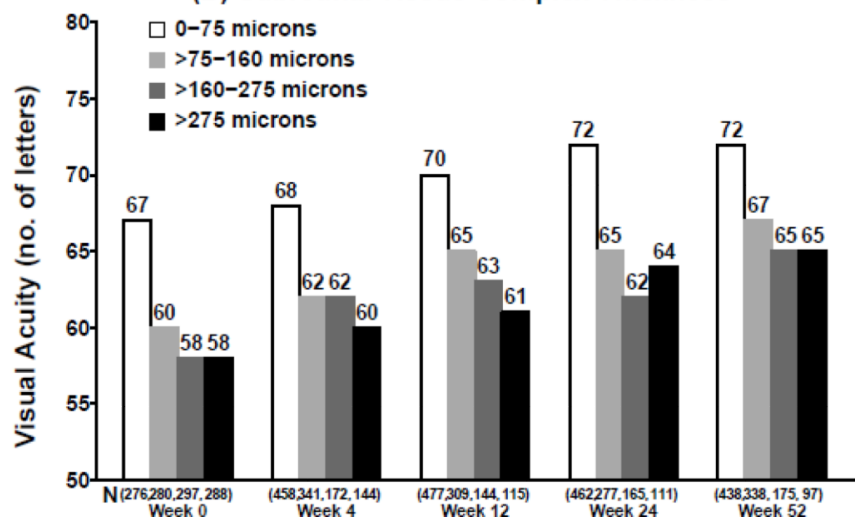

Figure 7.

Relationship between retinal thickness and visual acuity at baseline and followup. (A) foveal total thickness; (B) retinal thickness; (C) subretinal thickness; and (D) subretinal tissue complex thickness. 
(A) Foveal Total Thickness

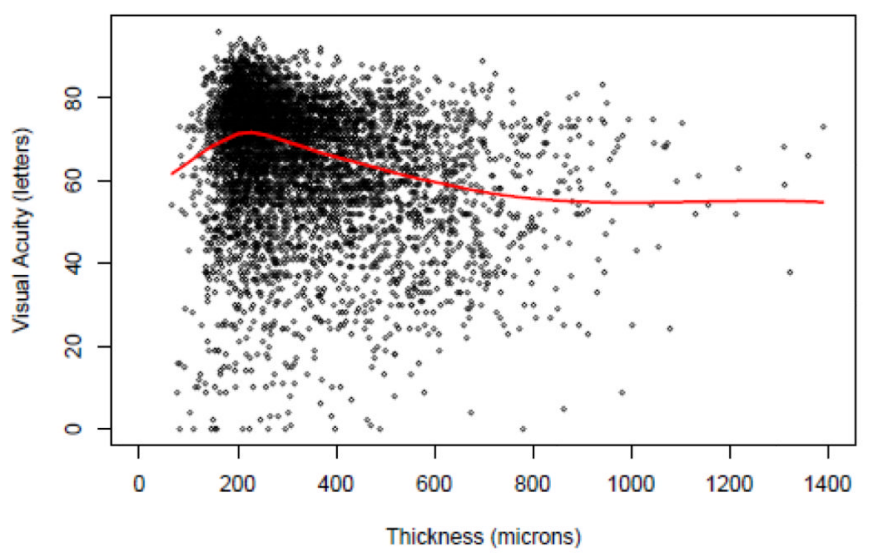

(C) Subretinal Thickness

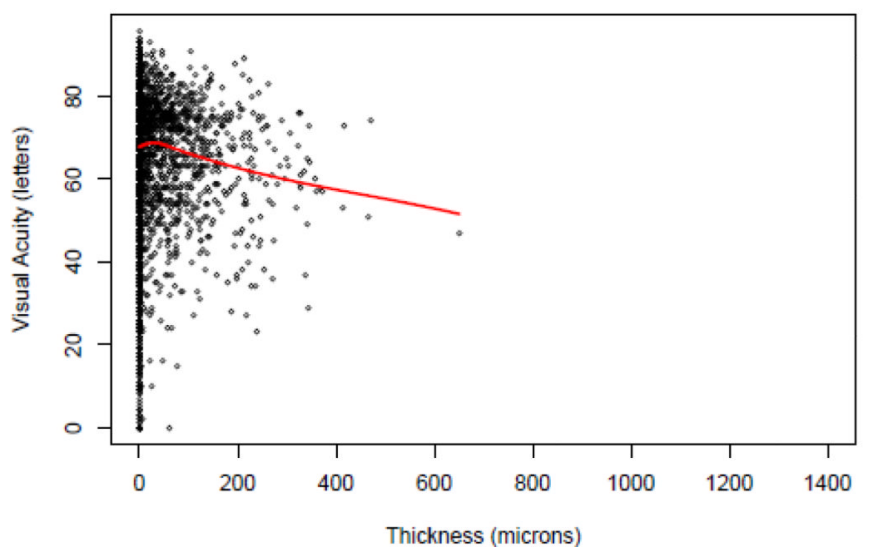

(B) Retinal Thickness

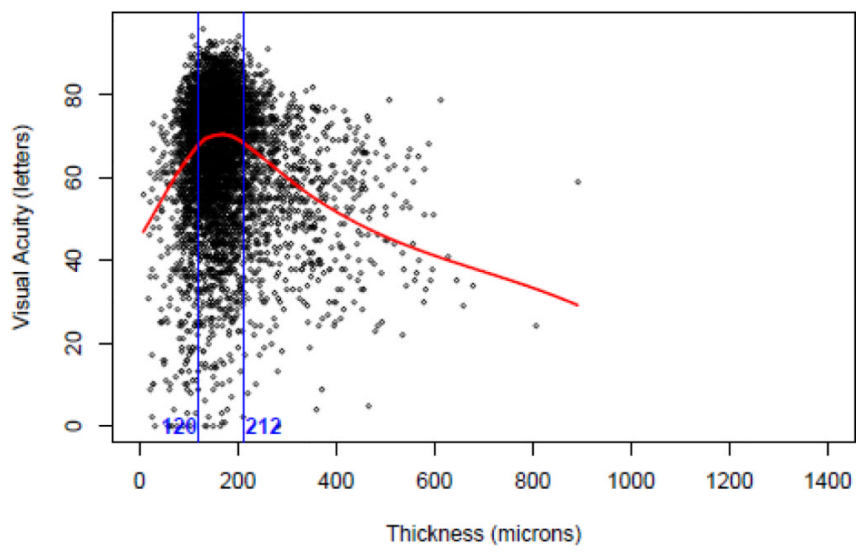

(D) Subretinal Tissue Complex Thickness

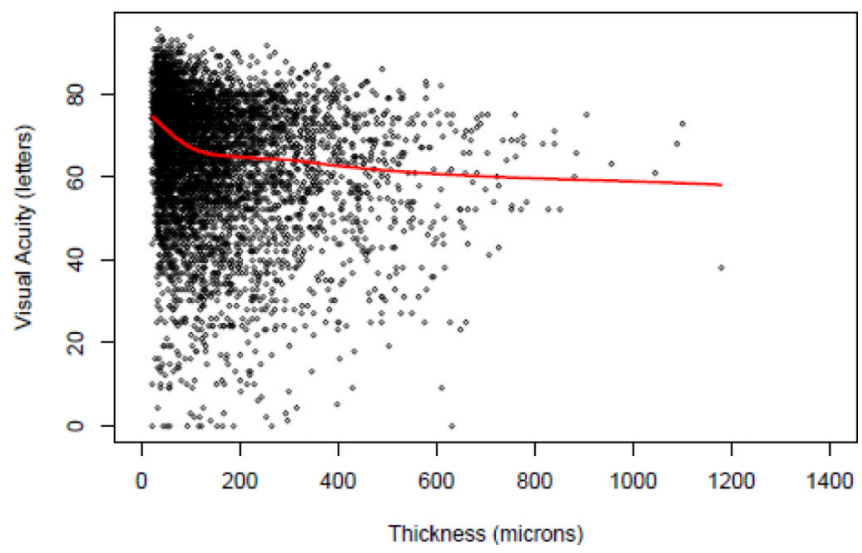

Figure 8.

Nonlinear relationship during follow-up of visual acuity with (A) foveal total thickness; (B) retinal thickness; (C) subretinal thickness; and (D) subretinal tissue complex thickness. 


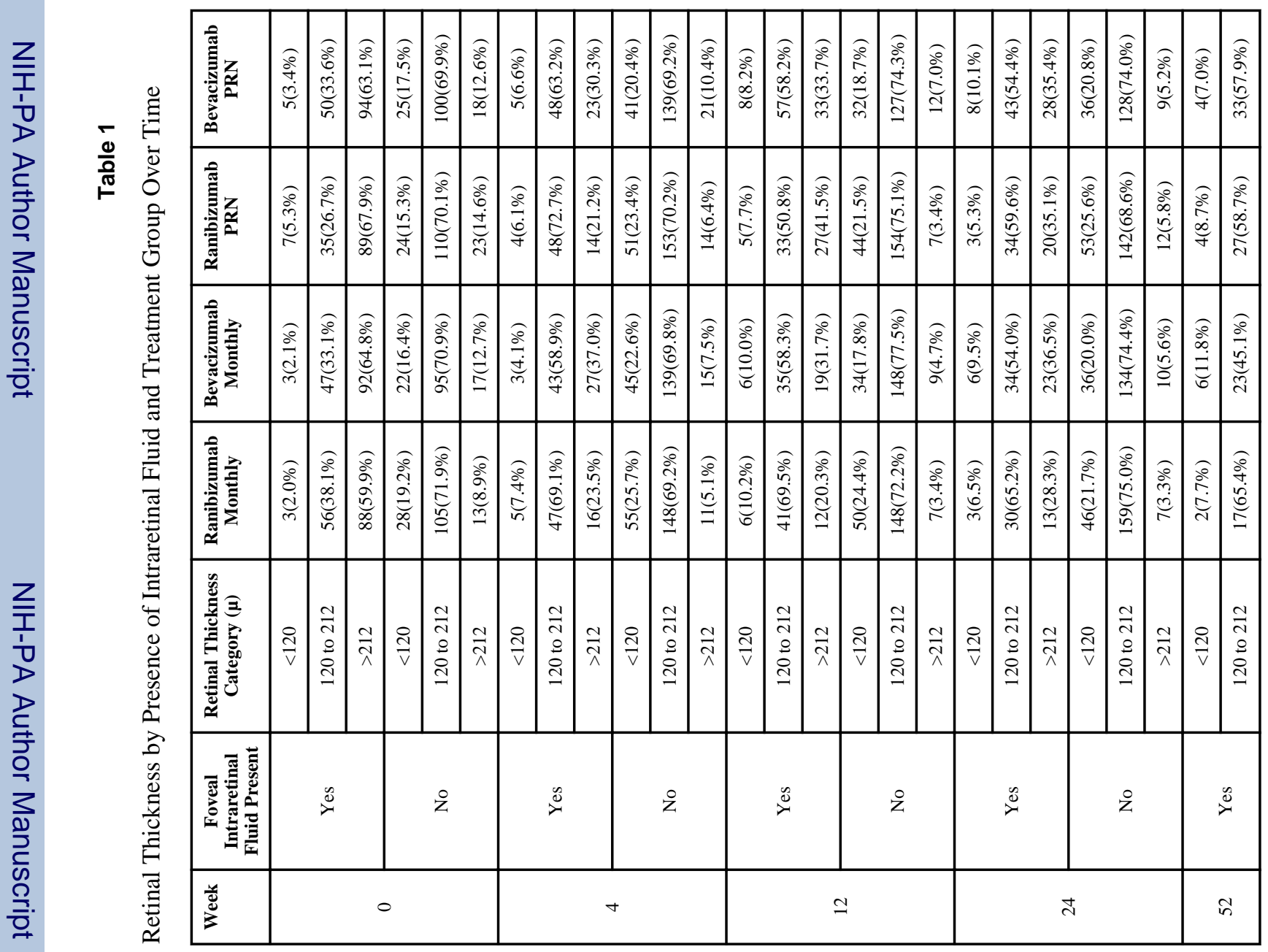




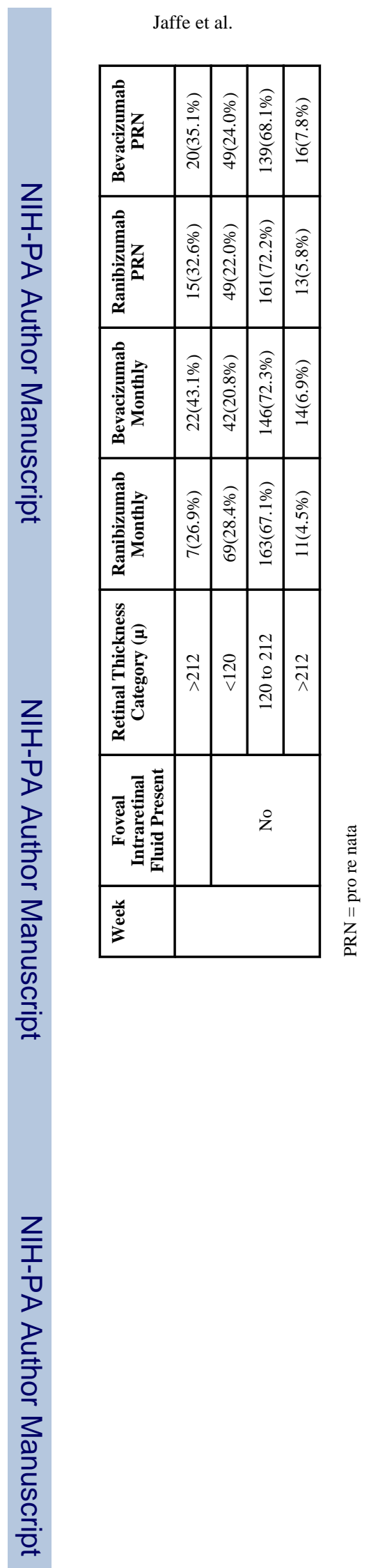

Ophthalmology. Author manuscript; available in PMC 2014 September 01. 
Table 2

Mean Visual Acuity at Week 52 by Neovascular Lesion Area and Pathology in the Foveal Center at Week 52 $(\mathrm{N}=1053)$

\begin{tabular}{|l|c|c|c|}
\hline Fundus Feature at Week 52 & N & $\begin{array}{c}\text { Unadjusted Mean } \\
\text { Visual Acuity Score } \\
\text { (Standard Error) }\end{array}$ & P values* \\
\hline Neovascular lesion area $\left(\mathrm{mm}^{2}\right)$ & & & $<0.0001$ \\
\hline$\searrow$ to $₫ .92$ & 244 & $74.3(1.11)$ & \\
\hline$>1.92$ to $₫ 4.96$ & 246 & $70.4(1.10)$ & \\
\hline$>4.96$ to $₫ .62$ & 245 & $67.1(1.10)$ & \\
\hline$>9.62$ & 242 & $61.9(1.11)$ & \\
\hline Missing & 76 & $63.1(1.98)$ & \\
\hline Pathology in foveal center & & & $<0.0001$ \\
\hline None & 202 & $73.9(1.20)$ & \\
\hline Fluid only & 85 & $75.3(1.85)$ & \\
\hline Choroidal neovascularization or serous pigment epithelium detachment & 259 & $69.7(1.06)$ & \\
\hline Non-geographic atrophy & 151 & $66.5(1.39)$ & \\
\hline Geographic atrophy, hemorrhage, RPE $\mathcal{\xi}$ tear, blocked fluorescence & 72 & $64.8(2.01)$ & \\
\hline Scar & 188 & $59.5(1.25)$ & \\
\hline Other ${ }^{\text {or missing }}$ & 96 & $66.8(1.75)$ & \\
\hline
\end{tabular}

* 1-way analysis of variance

$\mathrm{RPE}=$ retinal pigment epithelium

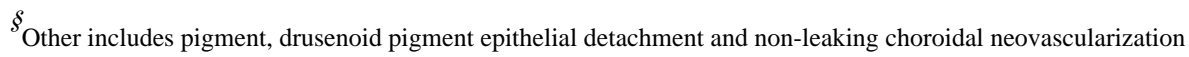


Table 3

Adjusted Mean Visual Acuity for Ocular Coherence Tomography and Fundus Features at Week 52(N=1004)*

\begin{tabular}{|c|c|c|c|}
\hline $\begin{array}{l}\text { Ocular Coherence Tomography } \\
\text { and Fundus Features at Week } 52\end{array}$ & $\mathbf{N}$ & $\begin{array}{l}\text { Adjusted Mean (SE) Visual } \\
\text { Acuity Score at Week 52 }\end{array}$ & P-value \\
\hline Intraretinal fluid & & & $<0.0001$ \\
\hline No Fluid & 527 & $70.9(0.68)$ & \\
\hline Fluid not in fovea center & 311 & $68.7(0.88)$ & \\
\hline Fluid in fovea center & 166 & $62.3(1.27)$ & \\
\hline Subretinal fluid & & & 0.02 \\
\hline No Fluid & 693 & $67.8(0.61)$ & \\
\hline Fluid not in fovea center & 156 & $71.7(1.29)$ & \\
\hline Fluid in fovea center & 155 & $70.4(1.29)$ & \\
\hline Retinal thickness $(\mu)$ & & & $<0.0001$ \\
\hline$<120$ & 216 & $60.9(1.10)$ & \\
\hline 120 to 212 & 680 & $71.1(0.60)$ & \\
\hline$>212$ & 108 & $70.2(1.59)$ & \\
\hline $\mathrm{CNV}$ lesion area $\left(\mathrm{mm}^{2}\right)$ & & & $<0.0001$ \\
\hline$>=0$ to $<=1.92$ & 239 & $72.4(1.12)$ & \\
\hline$>1.92$ to $<=4.96$ & 235 & $71.5(1.02)$ & \\
\hline$>4.96$ to $<=9.62$ & 234 & $69.1(1.04)$ & \\
\hline$>9.62$ & 226 & $64.4(1.08)$ & \\
\hline Missing & 70 & $61.0(2.21)$ & \\
\hline Pathology in the fovea center & & & $<0.0001$ \\
\hline None & 198 & $71.6(1.20)$ & \\
\hline Fluid only & 84 & $72.7(1.74)$ & \\
\hline Choroidal neovascularization or serous pigment epithelial detachment & 250 & $69.2(1.07)$ & \\
\hline Non-geographic atrophy & 142 & $69.1(1.34)$ & \\
\hline Geographic atrophy, hemorrhage, RPE tear, blocked fluorescence & 67 & $66.4(1.90)$ & \\
\hline Scar & 176 & $62.2(1.18)$ & \\
\hline Other or missing & 87 & $72.6(1.98)$ & \\
\hline
\end{tabular}

Subjects ( $\mathrm{n}=49)$ with missing data for fluid or retinal thickness were excluded.

$\mathrm{CNV}=$ choroidal neovascularization

$\mathrm{RPE}=$ retinal pigment epithelium; $\mathrm{SE}=$ standard error 